\title{
PRODUÇÃO CIENTÍFICA DE ENFERMAGEM NA REGIÃO NORDESTE (1988-1992)*
}

\author{
Marluce Maria Araújo Assis (Coordenadora)** \\ Maria Angela Alves do Nascimento** \\ Maria Salete Bessa Jorge ${ }^{\star \star \star}$ \\ Maria Socorro Matos ${ }^{\star \star \star \star}$

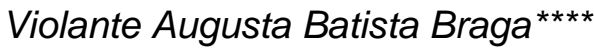

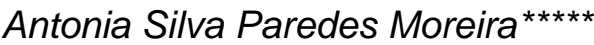 \\ Telma Ribeiro Garcia
}

Estudo exploratório descritivo realizado com o objetivo de identificar a produção científica das enfermeiras da Região Nordeste no período de 1988 a 1992, verificando o tipo de trabalho elaborado, as áreas temáticas predominantes e as formas de divulgação utilizadas. Observamos estar ocorrendo um aumento sensível nesta produção a partir de 1990. Os artigos científicos e a área temática assistencial predominaram, assim como a divulgação através de eventos nacionais.

UNITERMOS: produção científica de enfermagem, produção científica de enfermagem na região nordeste.

\footnotetext{
*Trabalho orientado pela Profa. Dr. ${ }^{a}$ Maria Cecília Puntel de Almeida - Professora Titular da Escola de Enfermagem de Ribeirão Preto da Universidade de São Paulo, Departamento de Enfermagem Materno-Infantil e Saúde-Pública.

${ }^{\star \star}$ Enfermeiras - Docentes da Universidade Estadual de Feira de Santana - Bahia. Mestre em Enfermagem de Saúde Pública e Doutoranda em Enfermagem na Escola de Enfermagem de Ribeirão Preto da Universidade de São Paulo, respectivamente.

***Enfermeira - Docente da Universidade Estadual do Ceará. Doutoranda em Enfermagem na Escola de Enfermagem de Ribeirão Preto da Universidade de São Paulo.

****Enfermeiras - Docentes da Universidade Federal do Ceará. Mestre em Enfermagem de Saúde Pública e Doutoranda em Enfermagem na Escola de Enfermagem de Ribeirão Preto da Universidade de São Paulo, respectivamente.

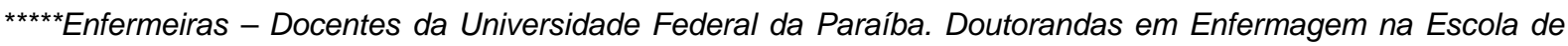
Enfermagem de Ribeirão Preto da Universidade de São Paulo.
} 


\section{INTRODUÇÃO}

O desenvolvimento da pesquisa em enfermagem no Brasil tem sido estudado a partir de alguns marcos históricos de referência, reconhecidos nacionalmente e que foram relatados entre outros autores por WRIGHT; PAIM; RODRIGUES (1983); BURLAMARQUE (1987) e MENDES (1991).

O primeiro desses marcos históricos é o levantamento de recursos e necessidades de enfermagem no Brasil, estudo realizado no período de 1956 a 1958 por um grupo de trabalho da Associação Brasileira de Enfermagem (ABEn), sob os auspícios da Fundação Rockfeller e o apoio do Ministério da Educação e Cultura (MEC), Fundação de Serviços Especiais de Saúde Pública (FSESP), Ministério da Saúde (MS) e Instituto Brasileiro de Geografia e Estatística (IBGE). (CARVALHO, 1976).

O segundo marco é a defesa da primeira tese de enfermagem por Glete de Alcântara, em 1963, para obtenção do titulo de professor catedrático da Universidade de São Paulo - Escola de Enfermagem de Ribeirão Preto.

O terceiro marco é o XVI Congresso de Enfermagem, realizado em 1964, ocasião em que o debate sobre pesquisa em enfermagem ocupou lugar de destaque na programação, sendo Enfermagem e Pesquisa o primeiro tema oficial do evento.

O quarto marco é representado pelo ingresso da enfermagem, no inicio da década de setenta, no sistema nacional de pós-graduação, por forca da Lei da Reforma Universitária (Lei 5540/68), que impôs mudanças no sistema educacional do país, entre as quais a exigência da redefinição do corpo docente a partir da qualificação acadêmica de mestres e doutores.

O quinto marco é a criação, também na década de setenta, do Centro de Pesquisa em Enfermagem (CEPEn), órgão da ABEn destinado a incentivar as investigações cientificas da profissão e responsável, entre outras atividades, pela realização dos Seminários Nacionais de Pesquisa em Enfermagem e pela elaboração e publicação de catálogos sobre pesquisas e pesquisadores de enfermagem no Brasil, onde são classificadas todas as dissertações e teses da enfermagem brasileira.

Dentre estes cinco marcos históricos de referência, o que trouxe mais repercussões efetivas para o desenvolvimento da produção científica da profissão foi, sem sombra de dúvidas, o ingresso da enfermagem no sistema nacional de pós-graduação, com a criação dos primeiros cursos de Mestrado em Enfermagem. No período de 1972-1979 foram implantados nove cursos em vários pontos do país, objetivando ampliar os níveis de competência e, as habilidades profissionais, e incentivar o desenvolvimento da pesquisa através da preparação de pesquisadores.

A maior concentração desses cursos está, até hoje, localizada na Região Sudeste, mais especificamente nos estados de São Paulo e Rio de Janeiro. Na Região 
Nordeste foram implantados em 1979, o Mestrado em Enfermagem Médico-Cirúrgica na Universidade Federal da Bahia (UFBA) e o Mestrado de Enfermagem de Saúde Pública na Universidade Federal da Paraíba (UFPB). Em 1993, quatorze anos depois dos cursos da UFBA e UFPB, é criado o Mestrado em Enfermagem de Saúde Comunitária da Universidade Federal do Ceará (UFCE).

A partir da década de oitenta, com o início da "abertura política" instalada na América Latina e no Brasil, ocorrem discussões ampliadas sobre as diversas tendências do pensamento expressas na pesquisa em enfermagem. Neste sentido, a produção cientifica da enfermagem torna-se objeto de estudo de várias enfermeiras que a analisaram sob diferentes perspectivas. Dentre esses trabalhos podemos citar o de VIEIRA (1980), que a estuda, no Brasil, no período de 1960 a 1979, verificando haver um evidente crescimento da mesma, principalmente a partir da segunda metade da década de sessenta; o de ALMEIDA et al. (1981), que analisa a produção do conhecimento em enfermagem ligada a pósgraduação, classificando as linhas de pesquisa nela privilegiada e o de BACHION et al. (1992), que a estuda, relacionada a pós-graduação de enfermagem no Brasil, no período de 1962 a 1991, através de catálogos do CEPEn.

Quanto ao conhecimento de enfermagem produzido na Região Nordeste (NE), ou tem sido incluído na análise geral de estudos realizados ao nível nacional, como citados anteriormente ou em estudos específicos de determinados estados da Região, como o de ROSENDO; ALBUQUERQUE (1992) para o estado de Alagoas, o de GARCIA; NÓBREGA (1993) para o estado da Paraíba; e o de BARROSO; VARELA; COSTA (1992) para o estado do Ceará ou ainda, enfocam o comportamento do mesmo em áreas específicas. Citamos como exemplo, a área de saúde da mulher e da criança contemplada em estudos de FERREIRA et al. (1990) sobre a situação da pesquisa em escolas de enfermagem da Bahia e o de SOBREIRA et al. (1992) sobre pesquisa e pesquisadores nesta mesma área na UFCE. Englobando a produção científica de todos os estados do Nordeste nenhum estudo ainda havia sido realizado.

Partindo dessas considerações, o presente estudo tem como objetivo:

Identificar a produção científica das enfermeiras da Região Nordeste, no período de 1988 a 1992, qual o tipo de trabalho desenvolvido, a tendência temática predominante e como tem sido divulgada essa produção.

Trata-se, portanto, nesta primeira aproximação, somente de um levantamento quantitativo que merecerá, em outros trabalhos posteriores, o estudo de questões outras ligadas à produção científica desta região do país.

\section{MATERIAL E MÉTODO}

Este estudo foi exploratório descritivo e definimos como "produção científica" trabalhos resultantes ou não de pesquisa, elaborados sob a forma de artigos científicos (relatórios de pesquisa, relatórios técnico-científicos, relatos de experiência, estudos de casos, dentre outros), resenhas, notas prévias, manuais de procedimentos ou de orientações, livros, capítulos de livros, teses, dissertações e monografias. 
Para obtenção dos dados foram utilizadas duas estratégias: um levantamento da produção científica elaborada nos cursos de graduação e pós-graduação em enfermagem através das escolas de Enfermagem da região Nordeste e um levantamento da produção científica divulgada no período de 1988 a 1992 em anais (XL a XLIV Congressos Brasileiros de Enfermagem, I Seminário Sul-Americano de Pesquisa em Enfermagem nas Áreas da Saúde da Mulher e da Criança - 1990 -, I,II e III Seminários Brasileiros de Comunicação em Enfermagem - 1988, 1990 e 1992 - V e VI Seminários Nacionais de Pesquisa em Enfermagem - 1988, 1991, I Seminário Internacional sobre AIDS: Um desafio para a Enfermagem - 1991 -, II Simpósio Nacional sobre Diagnostico de Enfermagem - 1992 -; Catálogos: (V, VI, VII, VIII, IX e X do Centro de Estudos e Pesquisas em Enfermagem); Periódicos (Revista Brasileira de Enfermagem, Revista Baiana de Enfermagem, Revista da Escola de Enfermagem da Universidade de São Paulo, Revista Enfoque, Revista Paulista de Enfermagem e Revista Saúde em Debate) e Programas (LX a LXIV Congressos Brasileiros de Enfermagem e do Encontro Internacional - "Pesquisa em Enfermagem: Uma questão de Saúde" - 1992).

O levantamento da produção científica elaborada nos cursos de graduação e pósgraduação foi feito através de formulário (Anexo 1 e 2) enviado para as 22 (vinte e duas) Escolas de Enfermagem da Região Nordeste, obtendo-se resposta de 13 (treze), o que representa um total de $59 \%$ das Escolas da Região e através do levantamento desta produção publicada em catálogos, periódicos, anais e programas de eventos científicoculturais. As Informações das Escolas sobre os trabalhos foram comparadas com os publicados, para evitar duplicação dos dados.

Para efeito do estudo, estes foram categorizados quanto a:

- Tipo de Trabalho: artigo científico (relatórios de pesquisa, relatórios técnicocientíficos, relatos de experiência, estudo de caso, dentre outros), resenhas, notas prévias, manual de procedimentos e livros, monografias, dissertações e teses.

- Área de Abrangência do Evento: internacional, nacional, regional e local.

- Fonte de Publicação: anais, periódicos, jornais e livros.

- Áreas Temáticas:

. Assistencial: trabalhos sobre sistematização das ações de enfermagem a pessoa, grupo ou comunidade (médico-cirúrgica, materno-infantil, psiquiatria, saúde pública e fundamental);

. Administrativa: trabalhos sobre planejamento, organização, direção, supervisão e controle da assistência de enfermagem; avaliação dos aspectos organizacionais da assistência; estudo sobre a avaliação das unidades de serviços de saúde, de enfermagem e auditoria;

- De Ensino: trabalhos relacionados ao ensino de enfermagem (auxiliar de enfermagem, técnico, graduação, especialização, mestrado e doutorado);

- Profissional: trabalhos sobre o objeto de trabalho da enfermagem, recursos humanos e mercado de trabalho, autonomia profissional, integração docente-assistencial, 
divisão social do trabalho, enfermagem e políticas de saúde, ética e legislação, produção, divulgação e utilização do conhecimento em enfermagem;

- De Reflexões Teóricas: trabalhos relacionados ao estudo, desenvolvimento de testagem de teorias e conceitos de enfermagem e outras áreas, elaboração e testagem de instrumentos.

Para a apresentação dos resultados obtidos distinguimos os trabalhos elaborados mas não divulgados; os apresentados em eventos científico-culturais; os publicados em anais, periódicos, jornais e livros; os elaborados ao nível de pós-graduação "stricto-sensu" e os monográficos elaborados ao nível de graduação, habilitação e especialização.

Tenha sido identificada ou através das respostas aos formulários enviados as Escolas de Enfermagem, ou da pesquisa nas fontes bibliográficas descritas no item anterior, essa produção foi categorizada conforme aplicável, segundo o tipo de trabalho, a área temática, a fonte de publicação e a abrangência do evento em que foram apresentados.

Para categorização das áreas temáticas, valemo-nos dos títulos dos trabalhos que foram informados pelos respondentes dos formulários enviados, o que representa uma limitação do estudo, dado que o titulo nem sempre é sugestivo ou indicativo do conteúdo. Quando o trabalho estava publicado além do título, o mesmo foi utilizado para esta classificação.

As monografias foram excluídas do processo de categorização, haja visto a diversidade de formas que as Escolas utilizaram para responder esse item do formulário. Algumas informaram apenas o quantitativo de monografias elaboradas por ano e nível de curso (graduação, habilitação e especialização); outras, além dessas informações listaram as elaboradas. Portanto as monografias foram apenas quantificadas.

\section{RESULTADOS E COMENTÁRIOS}

Na região NE há 22 Escolas de Enfermagem sendo que 13 fizeram parte do estudo. Destas, 11 são públicas (7 federais e 4 estaduais) e 2 particulares.

De 1988 a 1992, foram produzidos nesta região 1661 trabalhos assim discriminados: 836 (50,3\%) monografias elaboradas ao nível de graduação, habilitação e especialização; $542(32,6 \%)$ trabalhos apresentados em eventos científico-culturais; 160 (9,6\%) publicados; 68 (4,1\%) elaborados mas não divulgados e 55 (3,3\%) apresentados ao nível de pósgraduação "stricto-sensu" (dissertação de mestrado, teses de doutorado e livre-docência).

Quanto aos elaborados, mas não divulgados, são em número de 68: 04 (5,9\%) foram produzidos em 1988, 02 (2,9\%) em 1989; 09 (13,2\%) em 1990; 08 
$(11,8 \%)$ em 1991 e $45(66,2 \%)$ em 1992. 15 destes trabalhos, segundo as informações coletadas já foram aceitos para publicação encontrando-se no prelo.

Excluindo-se os monográficos (836 - 50,3\%) contamos com uma produção de 825 trabalhos. Destes, $542(65,7 \%)$ correspondem aos apresentados em eventos científicoculturais, 160 (19,4\%) publicados, 68 (8,2\%) elaborados e não publicados e 55 (6,6\%) elaborados ao nível de pós-graduação "stricto-sensu".

Do total de 68 trabalhos elaborados, mas não divulgados, 64 (94,1\%) foram classificados como artigos; 01 (1,5\%) como resenhas e $03(4,4 \%)$ como manuais de procedimentos. A área temática predominante foi a assistencial, com $41(60,3 \%)$ trabalhos nela classificados. Por ordem de freqüência seguem-se as áreas de reflexões teóricas, com 14 (20,6\%"); a área profissional, com 07 (10,3\%); a área de ensino, com 05 (7,3\%) e, por fim, a área administrativa, com apenas 01 (1,5\%).

A Tabela 1, apresentada a seguir, descreve os dados relativos aos trabalhos apresentados em eventos científico-culturais.

TABELA 1 - DISTRIBUIÇÃO DA PRODUÇÃO CIENTÍFICA DE ENFERMAGEM NA REGIÃO NORDESTE, QUANTO AO EVENTO, AO TIPO E A ÁREA TEMÁTICA DE TRABALHOS APRESENTADOS, NO PERÍODO DE 1988-1992

\begin{tabular}{|l|c|c|c|c|c|cc|}
\hline \multicolumn{1}{|c|}{ Especificação } & 1988 & 1989 & 1990 & 1991 & 1992 & TOTAL & $\%$ \\
\hline EVENTO & & & & & & & \\
Internacional & 3 & 3 & 9 & 29 & 15 & 59 & 10,9 \\
Nacional & 33 & 24 & 51 & 50 & 77 & 235 & 43,4 \\
Regional & 7 & 9 & 4 & 16 & 8 & 44 & 8,1 \\
Local & 24 & 25 & 25 & 63 & 67 & 204 & 37,6 \\
& & & & & & & \\
TIPO DE TRABALHO & & & & & & & \\
Artigo Científico & 67 & 56 & 83 & 157 & 156 & 519 & 95,7 \\
Resenha & - & - & 3 & - & 9 & 12 & 2,2 \\
Nota Prévia & - & 5 & 3 & - & - & 8 & 1,5 \\
Manual de & - & - & - & 1 & 2 & 3 & 0,6 \\
Procedimentos & & & & & & & \\
& & & & & & & \\
ÁREA TEMÁTICA & & & & & & & \\
Assistencial & 33 & 31 & 50 & 100 & 99 & 313 & 57,7 \\
Administrativa & 1 & 6 & 9 & 7 & 10 & 33 & 6,1 \\
Ensino & 7 & 7 & 11 & 15 & 9 & 49 & 9,1 \\
Profissional & 26 & 16 & 18 & 30 & 29 & 119 & 22,0 \\
Reflexões Teóricas & - & 1 & 1 & 6 & 20 & 28 & 5,1 \\
\hline TOTAL DE & 67 & 61 & 89 & 158 & 167 & & 542 \\
TRABALHOS & $(12,4 \%)$ & $(11,3 \%)$ & $(16,4 \%)$ & $(29,1 \%)$ & $(30,8 \%)$ & $(100,00 \%)$ \\
\hline
\end{tabular}


No período estudado foram divulgados em eventos científico-culturais 542 (65,7\%) da produção de 825 trabalhos. Deste total, $67(12,4 \%)$ trabalhos foram divulgados em 1988; 61 (11,3\%) em 1989; 89 (16,4\%) em 1990; 158 (29,1\%) em 1991 e 167 (30,8\%) em 1992. observa-se nesses dados estar havendo uma tendência ao aumento da motivação das enfermeiras para divulgarem os resultados de seus estudos e pesquisas, tendência esta que toma força a partir de 1990. Como estratégia para essa divulgação, deve-se ressaltar a promoção de eventos ao nível local e regional, que tem sido fomentado com maior freqüência. Dos 542 trabalhos, $253(43,4 \%)$ ocorreram em eventos realizados ao nível nacional; $204(37,6 \%)$ ao nível local; 59 (10,9\%) ao nível internacional e $44(8,1 \%)$ em eventos regionais. Estes dados podem ser visualizados na Figura 1.

\section{FIGURA 1 - Distribuição da produção científica de enfermagem na Região Nordeste, quanto à abrangência dos eventos científicos em que os trabalhos foram apresentados, no período de 1988-1992}

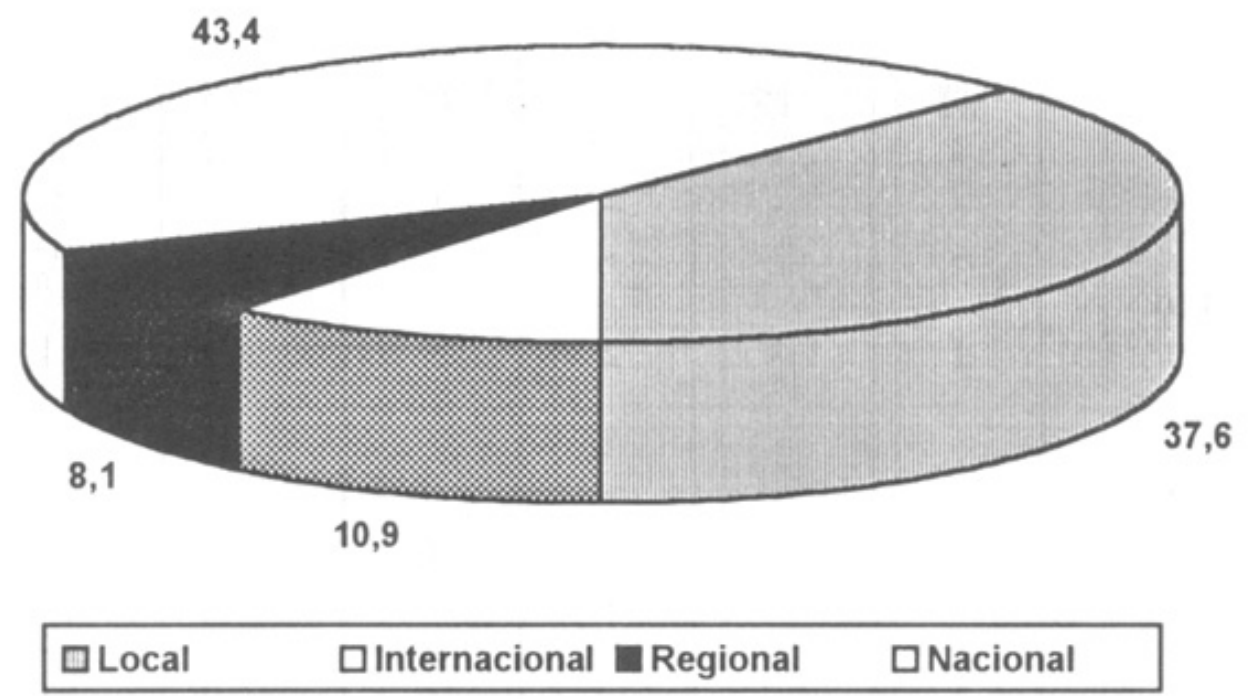

Quanto ao tipo de trabalho apresentado, 519 (95,7\%) foram classificados como artigos científicos; $12(2,2 \%)$ como resenhas; 08 (1,5\%) como notas prévias e $03(0,6 \%)$ como manuais de procedimentos.

A área temática predominante nestes trabalhos apresentados foi a assistencial, com $313(57,7 \%)$ trabalhos nela classificados. Por ordem de freqüência seguem-se as áreas profissionais, com 119 (22,0\%); a de ensino, com 49 (9,1\%); a área administrativa com 33 $(6,1 \%)$ e a área de reflexões teóricas, com 28 (5,1\%). 
Conclui-se que a enfermagem ainda utiliza como principal veiculo de divulgação de suas investigações, a comunicação em eventos (65,7\%) mesmo sendo esta uma forma de divulgação que não garante que o conhecimento gerado pelas mesmas, alcance toda comunidade científica da área.

Na Tabela 2, estão descritos os dados referentes aos trabalhos publicados.

TABELA 2 - DISTRIBUIÇÃO DA PRODUÇÃO CIENTÍFICA DE ENFERMAGEM NA REGIÃO NORDESTE, QUANTO A FONTE, O TIPO E A ÁREA TEMÁTICA DE TRABALHOS PUBLICADOS, NO PERÍODO DE 1988-1992

\begin{tabular}{|l|c|c|c|c|c|cc|}
\hline \multicolumn{1}{|c|}{ Especificação } & 1988 & 1989 & 1990 & 1991 & 1992 & TOTAL & $\%$ \\
\hline FONTE DE & & & & & & & \\
PUBLICAÇÃo & 8 & 1 & 7 & 7 & 20 & 43 & 26,9 \\
Anais & 13 & 8 & 26 & 21 & 30 & 98 & 61,3 \\
Periódicos & - & 3 & 1 & 2 & 2 & 8 & 5,0 \\
Jornais & 1 & - & 2 & 5 & 3 & 11 & 6,8 \\
Livros & & & & & & & \\
& & & & & & & \\
TIPO DE TRABALHO & 21 & 12 & 34 & 29 & 49 & 145 & 90,6 \\
Artigo Científico & - & - & - & - & 3 & 3 & 1,9 \\
Resenha & - & - & - & 1 & - & 1 & 0,6 \\
Nota Prévia & - & - & - & - & - & - & \\
Manual de & 1 & - & 2 & 5 & 3 & 11 & 6,8 \\
Procedimentos & & & & & & & \\
Livro & & & & & & & \\
& & & & & & & \\
ÁREA TEMÁTICA & 15 & 8 & 15 & 16 & 23 & 77 & 48,1 \\
Assistencial & - & 1 & 1 & 1 & 4 & 7 & 4,4 \\
Administrativa & 6 & 3 & 8 & 5 & 7 & 29 & 18,1 \\
Ensino & 1 & - & 11 & 10 & 10 & 32 & 20,0 \\
Profissional & - & - & 1 & 3 & 11 & 15 & 9,4 \\
Reflexões Teóricas & 22 & 12 & 36 & 35 & 55 & & 160 \\
\hline TOTAL DE & $(13,7 \%)$ & $(7,5 \%)$ & $(22,5 \%)$ & $(29,1 \%)$ & $(34,4 \%)$ & $(100,00 \%)$ \\
TRABALHOS & & & & & & \\
\hline
\end{tabular}

No período de 1988 a 1992, foram publicados 160 (19,4\%) trabalhos. Desse total, 98 $(61,3 \%)$ foram em periódicos; 43 (26,9\%) em anais; 08 (5,0\%) em jornais e 11 (6,8\%) como livros. Em sua grande maioria, estes trabalhos foram classificados como artigos científicos 145 (90,6\%); 03 (1,9\%) como resenha e 01 (0,6\%) como nota prévia. 
A temática predominante foi a assistencial com $77(48,1 \%)$ trabalhos nelas classificados. A área profissional foi a segunda mais freqüente, com 32 (20,0\%). Seguem-se a de ensino com $29(18,1 \%)$ trabalhos; a área de reflexões teóricas com $15(9,4 \%)$ e a administrativa com 07 (4,4\%).

A produção científica publicada representa uma porcentagem ainda pequena $(19,4 \%)$ do total de 825 investigações produzidas. Parcela significativa das mesmas tem sido publicada em periódicos locais. Caso não se dispusesse destes, esta produção poderia ser ainda menor.

A Tabela 3 apresenta os dados relativos aos trabalhos elaborados ao nível de pósgraduação "stricto-sensu".

TABELA 3 - DISTRIBUIÇÃO DA PRODUÇÃO CIENTÍFICA DE ENFERMAGEM NA REGIÃO NORDESTE, QUANTO AO TIPO E A ÁREA TEMÁTICA DE TRABALHOS ELABORADOS AO NÍVEL DE PÓS-GRADUAÇÃO "STRICTO SENSU", NO PERÍODO DE 1988-1992

\begin{tabular}{|c|c|c|c|c|c|c|c|}
\hline Especificação & 1988 & 1989 & 1990 & 1991 & 1992 & TOTAL & $\%$ \\
\hline \multicolumn{8}{|l|}{ TIPO DE TRABALHO } \\
\hline $\begin{array}{l}\text { Dissertação de } \\
\text { Mestrado }\end{array}$ & 10 & 14 & 7 & 10 & 8 & 49 & 89,1 \\
\hline Tese: & & & & & & & \\
\hline Doutorado & 2 & - & - & - & 2 & 4 & 7,3 \\
\hline Livre Docência & - & - & - & - & 2 & 2 & 3,6 \\
\hline Titular & - & - & - & - & - & - & \\
\hline \multicolumn{8}{|l|}{ ÁREA TEMÁTICA } \\
\hline Assistencial & 9 & 11 & 5 & 6 & 6 & 37 & 67,3 \\
\hline Administrativa & 1 & 1 & - & - & 2 & 4 & 7,3 \\
\hline Ensino & 3 & 2 & - & - & 1 & 6 & 10,9 \\
\hline Profissional & - & - & 2 & 4 & 2 & 8 & 14,5 \\
\hline Reflexões Teóricas & - & - & - & - & - & - & \\
\hline $\begin{array}{l}\text { TOTAL DE } \\
\text { TRABALHOS }\end{array}$ & $\begin{array}{c}13 \\
(23,6 \%)\end{array}$ & $\begin{array}{c}14 \\
(25,5 \%)\end{array}$ & $\begin{array}{c}7 \\
(12,7 \%)\end{array}$ & $\begin{array}{c}10 \\
(18,2 \%)\end{array}$ & $\begin{array}{c}11 \\
(20,0 \%)\end{array}$ & $\begin{array}{r}55 \\
(100,0\end{array}$ & \\
\hline
\end{tabular}

Do total de 55 trabalhos elaborados, no período de 1988-1992, ao nível de pósgraduação "stricto-sensu" por enfermeiras da região NE, 13 (23,6\%) o foram em 1988; 14 (25,5\%) em 1989; 07 (12,7\%) em 1990; 10 (18,2\%) em 1991 e 11 (20,0\%) em 1992. 
Quanto ao tipo de trabalho, $49(89,1 \%)$ foram realizados ao nível de mestrado; 04 $(7,3 \%)$ são teses de doutorado e $02(3,6 \%)$ de livre-docência. Observa-se que a produção de teses de doutorado é baixa o que é indicativo da demanda reprimida de docentes para este nível de qualificação.

A área temática predominante foi a assistencial com 37 (67,3\%) trabalhos, seguida da área profissional com 08 (14,5\%); a de ensino com 06 (10,9\%) e a administrativa com $04(7,3 \%)$ trabalhos nela classificados. Não houve estudos na área de reflexões teóricas, ao nível de pós-graduação "stricto-sensu", no período analisado.

A seguir passamos a fazer algumas considerações sobre a produção cientifica de forma geral.

Quanto ao período estudado, observa-se que tem havido um aumento dessa produção. Tomando-se os trabalhos realizados mas não divulgados (68), os apresentados em eventos científico-culturais (542), os publicados (160) e os executados ao nível de pósgraduação "stricto-sensu" (55), verifica-se que, no período de 1988 a 1992, foram produzidos 825 trabalhos na Região. Deste total, 106 (12,8\%) o foram em 1988; 89 (10,8\%) em 1989; 141 (17,1\%) em 1990; 221 (25,6\%) em 1991 e 278 (33,7\%) em 1992. Estas dados podem ser visualizados no Gráfico 1.

\section{GRÁFICO 1 - Distribuição geral da produção científica de enfermagem na Região Nordeste, no período de 1988-1992}

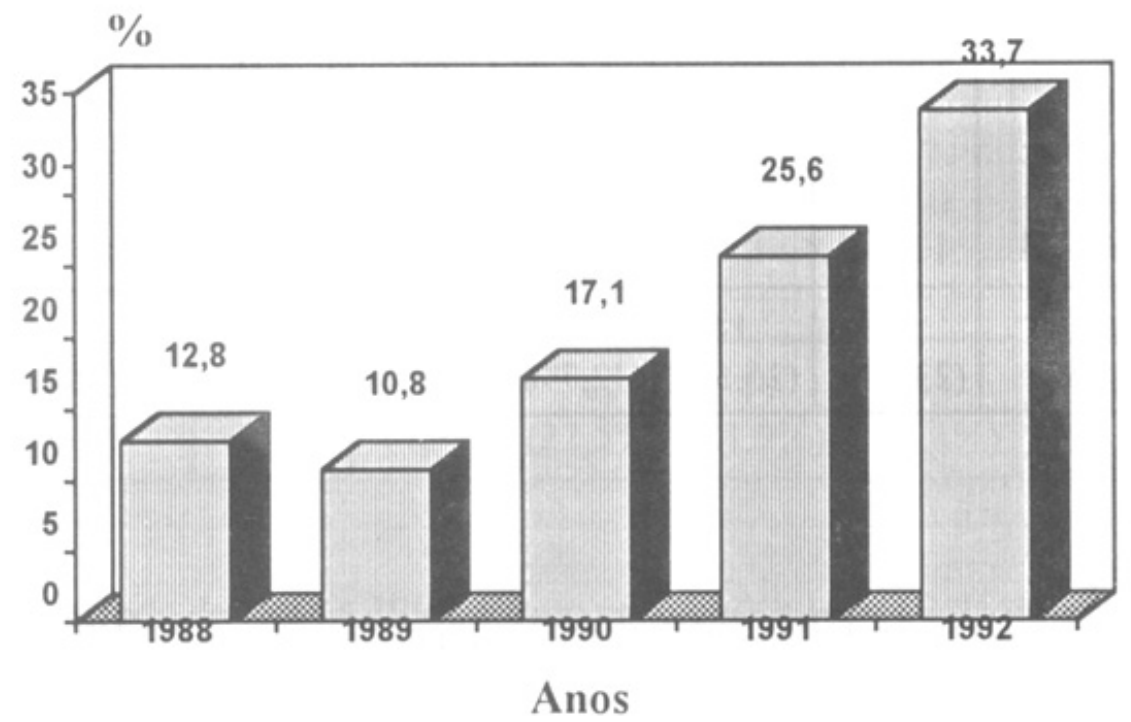


Levando-se em consideração que a maior parte dessa produção cientifica tem sido desenvolvida por enfermeiras docentes, podemos apontar como prováveis razoes para esse aumento no volume de trabalhos produzidos nos últimos anos: o estabelecimento nas instituições de ensino de qualificação e produção científica como critérios para avaliação de desempenhos e conseqüentemente progressão funcional e o incentivo salarial por produção cientifica de algumas instituições estaduais, a exemplo da Universidade Estadual de Feira de Santana (UEFS) e Universidade Estadual do Sudoeste da Bahia (UESB).

\section{GRÁFICO 2 - DISTRIBUIÇÃO DA PRODUÇÃO CIENTÍFICA DE ENFERMAGEM NA REGIÃO NORDESTE POR ESTADO, NO PERÍODO 1988- 1992}

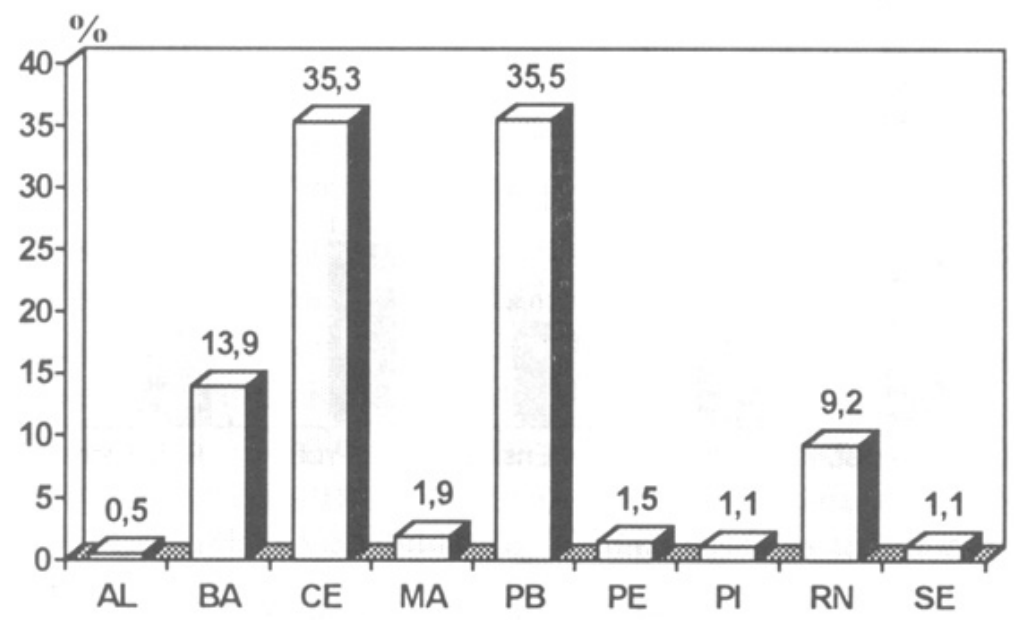

Legenda: AL - Alagoas; BA - Bahia; CE - Ceará; MA - Maranhão; PB - Paraiba; PE - Pernambuco; PI - Piaui; RN - Rio Grande do Norte; SE - Sergipe

O Gráfico 2 complementa a informação explicitada no primeiro, no que diz respeito a participação dos estados na produção científica da Região Nordeste. Vale ressaltar que esta participação, dos que apresentaram um percentual inferior a 2\%, não deve ser entendida como sua produção real. Provavelmente este percentual seria maior se as informações solicitadas houvessem sido devolvidas por todas as Escolas de Enfermagem consultadas, o que não ocorreu. Evidencia-se neste gráfico a elevada produção dos Estados do Ceará e da Paraíba, que são equivalentes. 
Merece destaque também o fato de que uma boa parte dos trabalhos elaborados pelas enfermeiras da Região são apresentados em eventos locais/regionais (ver Figura 1), o que dificultou o acesso aos programas e anais desses eventos e, portanto, à informação desejada.

\section{GRÁFICO 3 - DISTRIBUIÇÃO DA POPULAÇÃO CIENTÍFICA DE ENFERMAGEM NA REGIÃO NORDESTE, QUANTO AS ÁREASTEMÁTICAS, NO PERÍODO DE 1988-1992}

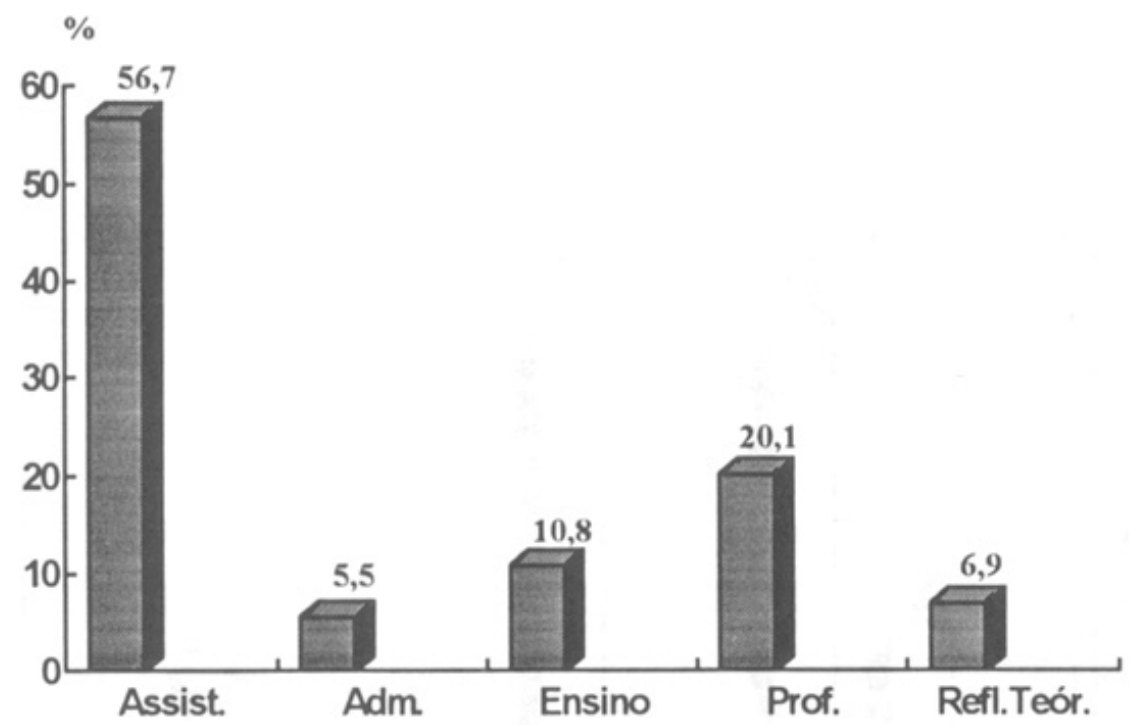

Quanto as áreas temáticas, observa-se no Gráfico 3, que há uma predominância da assistencial sobre as demais, acompanhando a tendência nacional já evidenciada nos trabalhos de ALMEIDA et al. (1981) e BACHION et al. (1992).

Chama a atenção o fato de que, a área profissional seja a segunda mais freqüente na produção cientifica da Região. Como possível explicação para esta ocorrência supomos que isto possa ser traduzido como maior engajamento das enfermeiras da Região Nordeste no processo de discussão de aspectos da política de saúde e sua participação em órgãos decisórios e gerenciais, além de uma maior preocupação que tenha ocorrido em analisar a enfermagem enquanto prática social.

Evidencia-se também, o fato de que, apesar da maior produção científica ser de enfermeiras docentes, somente $10,8 \%$ dos trabalhos estão relacionados à área de ensino.

Conforme explicado anteriormente, os trabalhos monográficos receberam tratamento distinto dada a não uniformização das informações enviadas. Assim, apresentamos somente o seu quantitativo por ano e nível de curso, na Tabela 4. 


\section{TABELA 4 - DISTRIBUIÇÃO DA PRODUÇÃO CIENTÍFICA DE ENFERMAGEM NA REGIÃO NORDESTE, QUANTO AO NÚMERO DE TRABALHOS MONOGRÁFICOS, NO PERÍODO DE 1988-1992}

\begin{tabular}{|l|c|c|c|c|c|cc|}
\hline \multicolumn{1}{|c|}{ Especificação } & 1988 & 1989 & 1990 & 1991 & 1992 & TOTAL & $\%$ \\
\hline TIPO DE TRABALHO & & & & & & & \\
NÍVEL: & & & & & & & \\
Graduação & 81 & 96 & 70 & 126 & 100 & 473 & 56,6 \\
Habilitação & 7 & 1 & - & - & - & 8 & 1,0 \\
Especialização & 87 & 48 & 70 & 57 & 93 & 355 & 42,4 \\
\hline TOTAL DE & 175 & 145 & 140 & 183 & 193 & \multicolumn{2}{c|}{836} \\
TRABALHOS & $(21,0 \%)$ & $(17,3 \%)$ & $(16,7 \%)$ & $(21,9 \%)$ & $(23,1 \%)$ & $(100,00 \%)$ \\
\hline
\end{tabular}

Do total de 836 trabalhos, 175 (21,0\%) foram produzidos em 1988; 145 (17,3\%) em 1989; 140 (16,7\%) em 1990; 183 (21,9\%) em 1991 e 193 (23,1\%) em 1992. Tais estudos foram distribuídos através dos níveis de graduação $(56,6 \%)$, habilitação $(1,0 \%)$ e especialização (42,4\%).

Vale destacar que o estado do Ceará apresenta 548 trabalhos, ou seja, 65,6\% do total de monografias da Região Nordeste.

Das 13 (treze) escolas estudadas, 05 (cinco) exigem investigação desta natureza na conclusão do curso de graduação, dentre as quais uma delas apenas a partir de 1992. com relação a habilitação, 02 (duas) escolas continuam oferecendo esta modalidade, das quais apenas 01 (uma) exige monografias no final do curso. Quanto a especialização, das 13 (treze) escolas, 08 (oito) tem curso de especialização, exigindo todas elas pesquisas monográficas.

\section{CONSIDERAÇÕES FINAIS}

Este estudo permitiu ter uma visão preliminar da produção científica de enfermagem da Região Nordeste, o que não havia sido realizado de forma distinta. Mesmo apresentando limitações seus resultados devem servir como ponto de partida para outros estudos desta região.

Observamos que houve elevação da produção científica ao longo do período estudado, representando este um percentual acumulado de 20,9\%.

. A área temática predominante foi a assistencial, seguida das áreas profissional e de ensino. 
. A área temática profissional, embora tenha ocupado o segundo lugar em termos de freqüência, destaca-se pelo interesse evidenciado em significativo número de pesquisas.

- Dos estados da Região Nordeste, verificamos que o da Paraíba e o do Ceará se destacam pela maior produção científica.

. Dos estados pesquisados, apenas duas Escolas de Enfermagem oferecem curso de pós-graduação a nível de mestrado (Bahia e Paraíba).

. A apresentação da produção científica ocorreu predominante em eventos nacionais e locais.

- A porcentagem de trabalhos publicados $(19,4 \%)$ ainda é baixa e as razoes prováveis são varias e dentre estas destacamos o restrito número de periódicos de enfermagem no Brasil, a reduzida quantidade de cursos de pós-graduação "stricto-sensu" na Região, a não existência do nível de doutorado também na Região e o pouco valor que as Universidades Federais davam a produção científica, como critério para progressões na carreira.

\section{NURSING SCIENTIFIC PRODUCTION IN NORTHEASTERN BRAZIL (1988-1992)}

Descriptive exploratory study accomplished with the purpose of identifying nurses' scientific productions in the Northeast Region during the period of 1988-1992, checking the rind of papers presented, the predominating theme area and the divulgation used. We observe that there is a sensitive increase in these productions since 1990. Scientific articles and assistencial area themes predominated, as did the divulgation trough national events.

UNITERMS: nursing scientific production, nursing scientific production in northeastern region.

\section{PRODUCCIÓN CIENTÍFICA DE ENFERMERÍA EN LA REGIÓN DEL NORDESTE (1988-1992)}

Estudio exploratorio descriptivo realizado con el objetivo de identificar la producción científica de las enfermeras de la Región Nordeste en el período de 1988 a 1992. Verificando el tipo de trabajo elaborado las áreas temáticas predominantes y las 
formas de divulgación utilizadas. Observamos que está ocurriendo un aumento sensible en esta producción a partir de 1990. Los artículos científicos y el área temática asistencial, predominaron, así como la divulgación a través de eventos nacionales.

UNITERMOS: producción científica de enfermería, producción científica de enfermería en la región nordeste.

\section{REFERÊNCIAS BIBLIOGRÁFICAS}

01. ALMEIDA, M. C. P. et al. A produção do conhecimento de pós-graduação em enfermagem no Brasil. In: CONGRESSO BRASILEIRO DE ENFERMAGEM, 23, 1981, Manaus. Anais. Manaus: Associação Brasileira de Enfermagem, 1981. p. 11927.

02. BACHION, M. M. et al. Um estudo sobre a pós-graduação de enfermagem no Brasil no período de 1962 a 1991. Ribeirão Preto: Escola de Enfermagem de Ribeirão Preto - Universidade de São Paulo, 1992. (mimeografado).

03. BARROSO, M. G. T., VARELA, K. M. de V., COSTA, L. B. Produção científica docente e discente do Departamento de Enfermagem - UFC: fatores condicionantes. Fortaleza, 1992. p. 10. (mimeografado).

04. BURLAMARQUE, C. S. Publicações e difusão do conhecimento em enfermagem no Brasil. Rev. Bras. Enfermagem. Brasília, v. 40, n. 4, p. 241-45, 1987.

05. CARVALHO, A. C. de. Associação Brasileira de Enfermagem - 1926/1976. Documentário. Rio de Janeiro: Folha Carioca Editora, 1976. p. 294-325.

06. FERREIRA, S. L. et al. Situação da pesquisa em saúde da criança nas Escolas de Enfermagem da Bahia, período 1980-1989. In: SEMINÁRIO SUL-AMERICANO DE PESQUISA EM ENFERMAGEM NAS ÁREAS DE SAÚDE DA MULHER E DA CRIANÇA, 1, 1990. Ribeirão Preto. Anais. Ribeirão Preto, 1990. p. 105-21.

07. GARCIA, T. R., NÓBREGA, M. M. L. Núcleos e linhas de pesquisa na produção científica da enfermagem da UFPB. João Pessoa, 1993. (mimeografado).

08. MENDES, I. A.C. Pesquisa em enfermagem: impacto na prática. São Paulo: EDUSP, 1991.

09. ROSENDO, C. A., ALBUQUERQUE, M. C. dos S. de. Aspectos da produção científica em Enfermagem na Universidade Federal de Alagoas. Maceió, 1992. (mimeografado). 
10. SOBREIRA, T. T. et al. Sumário bibliográfico sobre pesquisa e pesquisadores na área de saúde da mulher e da criança na UFCE (1985-1990). Fortaleza: Imprensa Universitária da UFCE, 1992.

11. VIEIRA, T. T. Produção científica em enfermagem no Brasil - 1960-1979. Salvador, p. 220. Tese (Livre Docência). Escola de Enfermagem - Universidade Federal da Bahia, 1980.

12. WRIGHT, M. da G. B., PAIM, L., RODRIGUES, K. H. Avaliação \& Perspectivas. Brasília: CNPq, 1983 (Ciências da Saúde, 6. Enfermagem, 38). 


\section{Anexo 1}

\section{PRODUÇÃO CIENTÍFICA DE ENFERMAGEM NA REGIÃO NORDESTE - 1988 A 1992 TRABALHOS MONOGRÁFICOS}

I. Instituição:

II. Entidade Mantenedora: Pública ( ) Particular ( ) Filantrópica ( )

1. Exige trabalho monográfico do aluno ao final do Curso de graduação em Enfermagem? $\operatorname{Sim}($ ) Não ( )

Em caso positivo, listar por ano (1988-1992), as monografias produzidas: nome do(s) autor(es) e titulo da monografia.

2. Ofereceu, no período de 1988 a 1992, Cursos de Habilitação em Enfermagem? $\operatorname{Sim}($ ) Não ( )

Em caso positivo, listar por ano (1988-1992), as monografias - nome do(s) autor(es) e título da monografia.

Ano(s) de oferecimento:

3. Exigiu trabalho monográfico do aluno ao final do Curso de Especialização?

$\operatorname{Sim}($ ) Não ( )

Em caso positivo, listar por ano (1988-1992), as monografias produzidas - nome do(s) autor(es) e titulo da monografia.

OBS: Caso não seja possível; listar as monografias, informar o seu quantitativo para cada um dos três casos acima, por ano de produção:

Exemplo: 1988 - Curso (Graduação, Habilitação ou Especialização) - número de monografias produzidas.

4. Oferece Curso de Mestrado?

$\operatorname{Sim}($ ) Não ( )

. Ano de Criação: 


\section{ANEXO 2}

\section{LEVANTAMENTO DA PRODUÇÃO CIENTÍFICA DE ENFERMAGEM NA REGIÃO NORDESTE, 1988-1992}

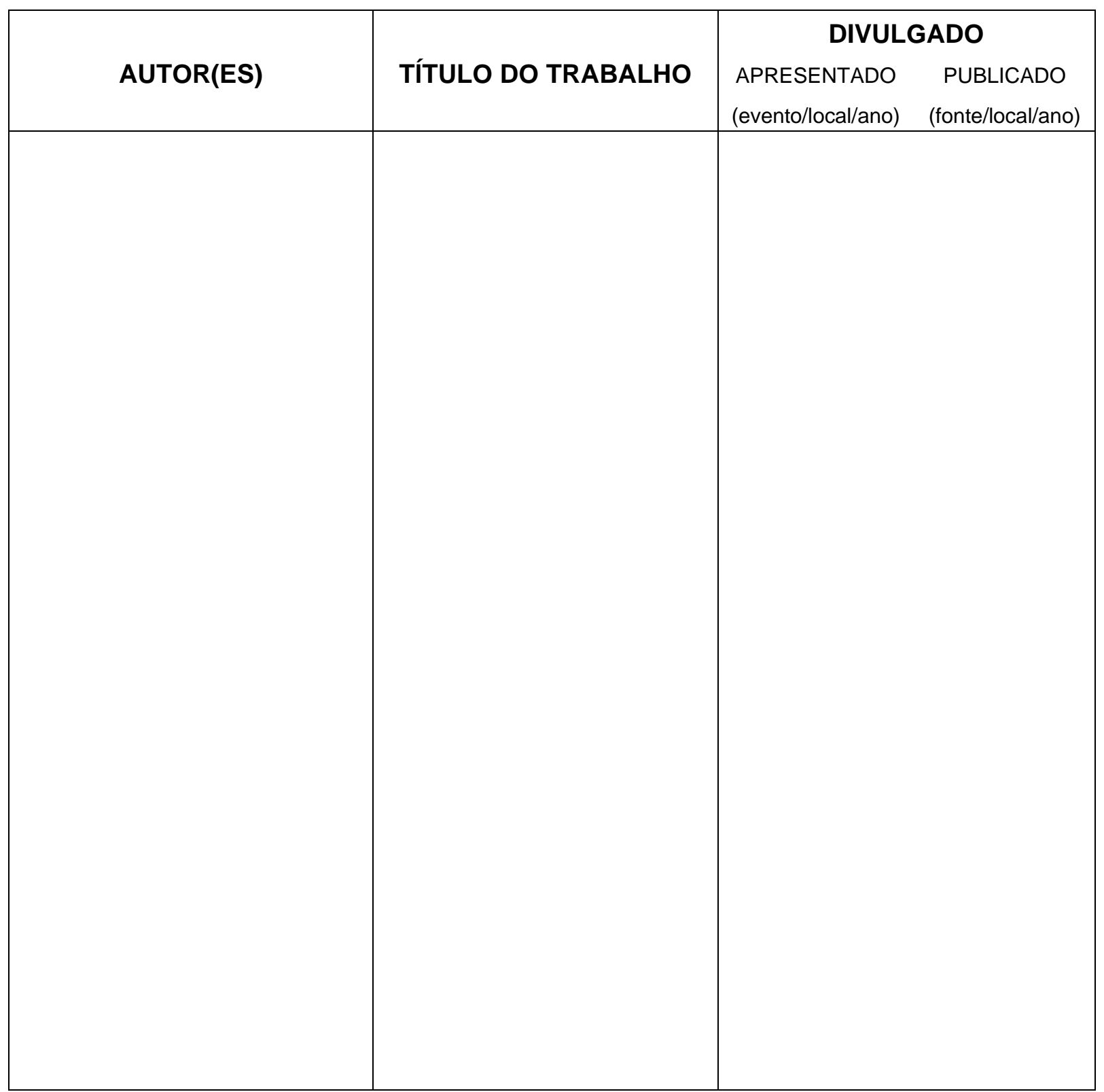

INSTRUÇÃO: Ex.: FREITAS, Maria. A Atuação do Enfermeiro na Saúde do Escolar. XX Congresso Brasileiro de enfermagem. Rev. Bras. de Enfermagem, 1990. Os trabalhos elaborados, mas não divulgados deverão também ser listados. 